\title{
Experimental load test statistics for the selected IPS tools on low-performance IoT devices
}

\author{
Tomas Zitta, Michal Lucki, Lukas Vojtech, \\ Marek Neruda, Lenka Mejzrova
}

\begin{abstract}
The goal of this paper is to propose a testing procedure for selected intrusion prevention systems (IPS) in a realistic network traffic in terms of their suitability on a given hardware microcomputers for low-performance devices for internet of things (IoT). We perform an IPS research in terms of resource usage in order to establish a universal procedure of checking, whether a given microcomputer controlling IoT devices (often in overloaded state) can additionally burden the installation and start-up of IPS. The experiment is repeated on several boards under overload condition to determine the maximum data rate, above which transmission degrades. The presented testing method is an exemplary tool for IoT applications concerning the security of embedded devices with low performance.
\end{abstract}

K e y w ord s: security, intrusion prevention systems, load analysis, low-power devices, IoT, horn method

\section{Introduction}

Currently, such intrusion detection systems as Snort or Suricata are well optimized for operation on powerful multi-core server stations. However, microcomputers, such as Raspberry $\mathrm{Pi}$, are often used to control monitor Internet of Things (IoT) devices and their traffic. Since the supervision process can be remote, the microcomputer should be protected, for example by some IPS tools. Considering the fact that microcomputers offer limited hardware resources and that they work continuously, very often under overload conditions, it is possible that installation and running another application could cause a slowdown or even stop some of the running processes. Thus, it is not obvious that running IPS on every microcomputer, for any traffic would be successful. Establishing a well-defined procedure of testing some selected IPS tools launched on a specific low-performance equipment as the Raspberry Pi platform could facilitate the decision-making, whether adding an IPS to a given hardware is reasonable and beneficial. The main test feature is measurements of RAM consumption and CPU load, aimed at reducing the consumption of system resources. An IoT system developer would then apply a similar test and decide, if Snort or Suricata can be run on a given IoT system, with some specific microcomputer, and under some specific traffic level.

The Introduction chapter discusses the main features of Snort and Suricata including selected evaluation criteria, and the new results are in the following chapters. We supported our claims by the measurements and this is our contribution and a step forward compared to the previous works. The goal is not to make a list of hardware configuration, but to propose a testing procedure for Snort/Suricata (as commonly used tools) in terms of their suitability to be employed as IPS for a given traffic and on a given hardware.

The successful development of the IoT services opens the debate on how to reduce operational costs, propose cost effective solutions and ensure environment-friendly operation. Satisfying hardware requirements related to computing power, energy consumption, creating space for new applications to run on current hardware, and deployment of a new firmware electronics for households. Another problem is protecting IoT devices against cybercrime; which includes intrusion detection and prevention against online threats [1]. Considering that IoT influences more and more applications and devices, a systematic approach to security is necessary in order to prevent attempts at manipulating or controlling IoT devices. IoT systems can encounter such threats during remote firmware or software upgrades. Individual security components within IoT concepts may also be subjected to attacks. One reason why IPSs should be used in telecommunications is that protection should be able to work with continuous traffic. The most advanced solutions include remote access, ability to work in a cloud environment, and efficient electric power scenarios [2][3][4]. These are just several reasons why data should be protected in an optimal way, while coping with significant data traffic and IPS, considering the fact that low-performance devices do not offer huge RAM and CPU margin.

\footnotetext{
* * Czech Technical University in Prague, Faculty of Electrical Engineering, Department of Telecommunication Engineering, Technická 2, 16627 Prague 6, Czech Republic tomas.zitta@fel.cvut.cz, michal.lucki@fel.cvut.cz, lukas.vojtech@fel.cvut.cz, marek.neruda@fel.cvut.cz, lenka.mejzrova@fel.cvut.cz
}

DOI: 10.2478/jee-2019-0058, Print (till 2015) ISSN 1335-3632, On-line ISSN 1339-309X

(C) This is an open access article licensed under the Creative Commons Attribution-NonCommercial-NoDerivs License (http://creativecommons.org/licenses/by-nc-nd/3.0/) 


\section{Related works}

Security systems offer tools for intrusion detection and prevention, which results in high implementation and maintenance costs. One potential solution is implementing integrated security tools. These tools should be installed on network nodes, such as routers or switches. This also requires advanced algorithms for optimal operation within a given topology. Potential intrusions can be treated by IPS. The main goal of such a system is to identify attacks based on adaptive matching algorithms that compare the threat with a reference pattern. They can be employed at the hardware or software level. The pattern-matching intrusion detection technique was discussed in [5]. Protection should include intrusion detection and prevention. It has been confirmed that matching identified patterns with those stored in a database is one of the most effective tools for this purpose. Alerts should be considered in cases of continuous mismatch between the patterns and significant increase in threats requires multistep security [5].

\subsection{Selected intrusion prevention systems}

There are several methods and software tools for IPS. One of the available software tools is Snort [6]. Snort collects traffic data, and monitors and analyzes the data. It is known for its open architecture. A number of rules were optimized in a Snort-based security tool, which included; protection against malware, online attacks, vulnerabilities, among others; by using a database of rules [7]. Additional rules may be implemented for adaptive adjustments based on the performance of Snort. The operation of Snort includes the analysis of protocols, and searching and matching content. As mentioned in [8], Snort can run in different modes of operation; such as sniffer, packet logger or network attack detector. The rules can be sent automatically based on data sent by a server. The main idea presented in [8] is that a server collects data and sends the data to Snort, which performs an evaluation to generate a rule automatically. The rule can then filter the acquired data. The performance analysis is the topic of [9], where it is stated that Snort works correctly if updated regularly, otherwise it will not be able to detect the newest types of attacks and threats. The operation of Snort can be enhanced for example by using dynamic fuzzy rule interpolation, which enhances Snort to current network conditions dynamically [10].

Another popular tool for IPS is Suricata. An attempt of an experimental comparison of Suricata and Snort is presented in [11]. It was stated that, while running on a high-performance machine (ie with a 12-core CPU, 125 $\mathrm{Gb}$ of RAM and CentOS 5.4. operating system), Suricata uses multi-threading to improve processing speed in comparison to Snort. The authors evaluated both tools in terms of memory requirements, speed and the accuracy of intrusion detection. The conclusion was that Suricatas performance was comparable to that of Snorts. However, the results do not include a statistical evaluation of the experiment. Furthermore, the investigation was inconclusive because of false alarms as well as ignored attacks; which are reported as disadvantages for Suricata. This is in contrary to our experiment, which was carried out on a low-performance machine, such as Raspberry Pi; where we proposed a well-defined, universal method for testing the load of IPS in low performance embedded IoT devices.

\subsection{Performance analysis}

Other significant papers dealing with a performance analysis of Snort and Suricata can be found in [12][13], focused on the performance rather than on the universal testing procedure in a given traffic. Let us discuss main features of the tested tools and the evaluation criteria. A sample list of evaluation criteria on security tests can be found in [14], where the metrics are: CPU load, number of packets lost, and number of alerts all measured under varied traffic rates, types of attacks, different rule sets, as well as for normal and malicious traffic. It was stated that each software acts in a different way for each attack, thus each exhibits different packets lost and different alerts. However, including a distinct set of rules in each IPS was found as a benefit to the overall security of the system. The emphasis is that both performance and accuracy should be considered while introducing new solutions. Other software tools can be used for this purpose [15]. In [16], it is claimed that the signature-based Snort is not suitable for advanced customization and high-speed networks because of its simplicity and low-end nature. Suricata is recommended as a multi-thread architecture that can cope well with anomalous situations and large-extent customization. This is at the price of significant CPU and memory use. This is also confirmed in [17], where the focus is related to the differences between the bit obsolete Windows Server 2008 and Linux. The main conclusion was that Suricata displays greater RAM and CPU usage, but performs better in terms of packets dropped due to threats. However, no graphs are presented to justify these claims. Both Snort and Suricata were able to identify all attacks correctly. Power consumption was one of the topics addressed in [18]. The authors proposed an adaptive multistep algorithm that managed to save power, which is a step towards environmental-friendly solutions.

Regarding the previously stated examples, one of the goals addressed in this paper is to establish the optimum set of parameters for hardware resources for effective intrusion detection and prevention in the selected software tools used in IoT. This includes efficient use of computing power in terms of CPU usage, use of RAM or bus for data handling. The testing method used in our research could be useful when comparing other IPS products in the future. The key findings are that CPU loads vary when employing Snort or Suricata, based on traffic, which requires network traffic models. Memory use, however, can be well estimated. The two commercially available software tools investigated for this purpose are Snort and Suricata. They are both implemented on the Raspberry Pi platform. 


\section{Methods}

Both Snort and Suricata are eligible to be used as IPS, however, either may be more suitable depending on conditions. The experiment includes measurements of system resources handling Transmission Control Protocol (TCP) and User Datagram Protocol (UDP) network traffic at a data rate of $5 \mathrm{Mbps}$. The measured data is evaluated using the Horn method [19]. The System Activity Report (SAR) tool is used to gather information about the system. The so-called Iperf tool is used to generate network traffic [20]. Iperf is a tool for network performance measurement, eg to measure maximum TCP bandwidth, allowing the tuning of various parameters and UDP characteristics. Iperf reports bandwidth, delay jitter, datagram loss. Typical Iperf output contains a time-stamped report of the amount of data transferred and the throughput measured. The data streams can be either TCP or UDP traffic. It consists of both a server and client side. The network traffic in this experiment includes one-way UDP and TCP traffic. The server is run on Raspberry Pi 3 (also Raspberry Pi 2 or 1 for the comparison purposes in the following experiments), and the client is run on a laptop computer. The experiment consists of 8 types of measurement for both Snort and Suricata. It has been proven that CPU load and RAM consumption are the major problems to be solved for optimal prevention in IoT networks. For this reason, a well-established method for testing the IPS tools is established. On average, Snort took up more than $100 \mathrm{MB}$ of memory in comparison to Suricata and exhibited CPU loads up to 5

\subsection{Performance analysis}

There are several methods that can be considered for IPS tests on traffic, and one of them is the Horn method [19]. The Horn method is used to determine the number of runs for each test to be significant. This method is adequate for analyzing small statistical samples, $i e$ between 4 and 20 samples, which perfectly meets our experiment. Claims created on the basis of small samples are potentially affected by greater uncertainty. The method is an analysis-based order of statistics, where one of the parameters is defined as depth related to the samples quantiles. The pivot depth and furthwer parameters, see [19], can be calculated as

$$
H=\operatorname{int} \frac{1}{2}\left[\frac{n+1}{2}\right], \quad H=\operatorname{int} \frac{1}{2}\left[\frac{n+1}{2}+1\right]
$$

where $n$ is the amount of samples in a data set from population. (In the Horn method, $n$ is usually in the range of $4<n<20$ ). The pivot point low $x_{L}$ and high $x_{H}$ can be obtained

$$
\begin{gathered}
x_{L}=x(H) \\
x_{H}=x(n+1-H)
\end{gathered}
$$

where $x_{H}$ is pivot point high, $x_{L}$ is pivot point low; the floor function that takes a real number as input and gives the greatest integer less than or equal to the argument

$$
x(i) \leqslant x(i+1)
$$

where $x(i)$ are ordered statistics, ie (5), [19]. The estimate of the parameter of location is pivot half sum $P_{L}$ will be

$$
P_{L}=\frac{x_{L}+x_{H}}{2}
$$

The estimation of spread parameter, pivot range $R_{L}$, is

$$
R_{L}=x_{H}-x_{L}
$$

Random variable $T_{L}$ has approximately symmetric distribution, whose chosen quantiles are

$$
T_{L}=\frac{P_{L}}{R_{L}}=\frac{x_{L}+x_{H}}{2\left(x_{H}-x_{L}\right)}
$$

$P_{L}, R_{L}$ and $T_{L}$ are dimensionless, since pivot half sum $P_{L}$ is a sum of two numerical values (divided by two), pivot range $R_{L}$ is difference of pivot points high and low that are numbers, too, and $T_{L}$ is their ratio. Since $\alpha$ is the probability of rejecting the hypothesis when it is true, for a given statistics $t$, the confidence interval $100(1-\alpha) \%$

$$
P_{L} \pm t_{1-\alpha / 2} \cdot R_{L} \equiv \frac{x_{L}+x_{H}}{2} \pm t_{1-\alpha / 2} \cdot\left(x_{H}-x_{L}\right)
$$

The $90 \%$ confidence interval of the mean value is

$$
P_{L}-R_{L} t_{L, 0.90}(n) \leqslant \mu \leqslant P_{L}+R_{L} t_{L, 0.90}(n)
$$

Results are expressed as the distance difference between the actual and estimated position. In our case, the $90 \%$ confidence interval and the number of tests being 7 , result in the quantile value equal to 0.451 , which lead to the correct estimation of the spread parameter. (A given confidence interval and the number of tests is associated with the specific quantile value).

\subsection{Testing methodology}

The experiment includes measurements of system resources handling by Snort and Suricata in terms of the CPU load and RAM usage. The SAR tool is used to gather information about the system. The Iperf tool is used to generate network traffic. Iperf consists of a server and a client. The network traffic used in this experiment is one-way UDP and TCP traffic. The server is run on Raspberry Pi 3, the client is run on a laptop computer (Acer Aspire V3-571G with Linux Mint 18.2 Cinnamon). A low-power computer, such as Raspberry $\mathrm{Pi}$ is chosen as a server hardware, based on the demand for commercial availability, low cost and low power consumption in embedded IoT devices. The microcomputer plays the role of a node in the IoT network doubled as an IDS probe. A full-fledged laptop is chosen to be the client, as commonly 


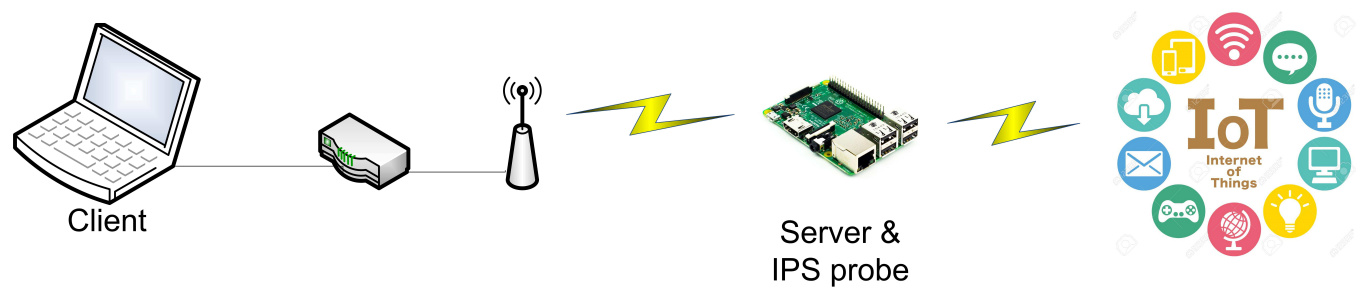

Fig. 1. Topology of experiment establishing the server-client connection for testing IPS run on a microcomputer

used in households to control IoT devices. The connection is established by using a Wi-Fi access point. The router was connected to the computer by Ethernet and Raspberry Pi via integrated Wi-Fi chip, Fig. 1. By using a conventional Wi-Fi router, we implement the IEEE $802.11 \mathrm{n}$ recommendation that offers $100 \mathrm{Mbps}$, comparable with Fast Ethernet (in theory, the maximum bitrate is $600 \mathrm{Mbps}$ ), $40 \mathrm{MHz}$ bandwidth (using multiple-input and multiple-output (MIMO) streams.

Table 1. Communication network traffic - UDP and TCP traffic for testing Snort and Suricata CPU load and RAM usage, Duration $120 \mathrm{~s}$

\begin{tabular}{|c|c|c|c|}
\hline & streams & $\begin{array}{r}\text { Streams } \\
(\mathrm{Mbps}) \\
\end{array}$ & $\begin{array}{c}\text { Bit rate } \\
(\mathrm{s})\end{array}$ \\
\hline TCP & $1448 \mathrm{~B}$ & 1 & 5 \\
\hline TCP & 1448 B & $10^{1)}$ & $0.5^{2)}$ \\
\hline $\mathrm{TCP}$ & $138 \mathrm{~B}$ & 1 & 5 \\
\hline UDP & $1468 \mathrm{~B}$ & 1 & 5 \\
\hline UDP & $1468 \mathrm{~B}$ & $10^{1)}$ & $0.5^{2)}$ \\
\hline UDP & $158 \mathrm{~B}$ & 1 & 5 \\
\hline
\end{tabular}

1) parallel, $^{2)}$ each

The experiment conceptualizes eight types of measurement. The following communication network traffic options were performed, which cover possible network traffic options (see Table 1). Each type of measurement is repeated seven times for statistical processing purposes, as required by the chosen quantile that equates to 0.451 . This allows estimating the spread parameter in Horns method. Each measurement begins 90 seconds after all boot processes of the Raspbian operating system are performed and takes 5 minutes to record and compare all necessary data. The Raspberry Pi 3 platform uses the Raspbian Stretch operating system. The specification ( $e g$ for reproducibility purposes) is presented in Table 2. The Raspbian is restarted after a measurement. The process is performed as follows:

- 0:00 Start of experiment, SAR commands are executed.

- 0:20 Snort or Suricata is started.

- 2:00 (120s) Iperfs transmission is started.

- 4:00 (240s) Iperfs transmission is completed.

- 4:40 (280s) Snort or Suricata is interrupted, only the system is running.

- 5:00 (300s) End of experiment.
Both Snort and Suricata are run in an IPS mode. The tested version of Snort is 2.9.9.0. The Snort is equipped with the registered version of VRT rules 2990, containing 31962 rules, but only 31942 Snort rules were actually used because of the compatibility with Suricata. Snort is configured to run in-line, using NFQ (a queuing type) for data acquisition. The tested version of Suricata is 4.0.0 with the same rule set as Snort. The compatibility of the VRT rules with Suricata is not complete since 40 rules are not compatible; therefore, 31922 Suricata rules were used. The rules were installed using the Oinkmaster tool. Suricata and Snort are both configured to run in IPS mode (Table 3). Iperf can modify the Maximum Segment Size (MSS) in TCP segments (20B Header + MSS). The size of MSS for TCP commands is larger since Iperf notifies the correct size in a terminal. To confirm the significance of the hardware platform evaluated, the mean difference values of CPU and RAM results were calculated for each communication network traffic. All calculated mean values are significantly different from zero, $i e$ the effect of used hardware platform is statistically significant for the observed CPU and RAM loads. The SAR tool is used to gather information on the system based on a count and an interval parameter (see Table 10 in Appendix). The information is saved in a file. SAR collects information on CPU and RAM usage during the experiment. Iperf is used to generate network traffic. The server side and the network traffic are started. The results of our experiment can be a useful guideline for testing similar low-performance devices.

Table 2. Hardware resources for testing the CPU load and RAM usage

\begin{tabular}{ll}
\hline Parameter & Specification \\
\hline SoC & Broadcom BCM2837 \\
CPU & 4 ARM Cortex-A53, 1.2GHz \\
GPU & Broadcom VideoCore IV \\
RAM & 1GB LPDDR2 (900 MHz) \\
Networking & 10/100 Ethernet \\
Bluetooth & 2.4 Classic $802.11 \mathrm{n}$ \\
Bluetooth & Low Energy \\
Storage & microSD \\
\hline
\end{tabular}




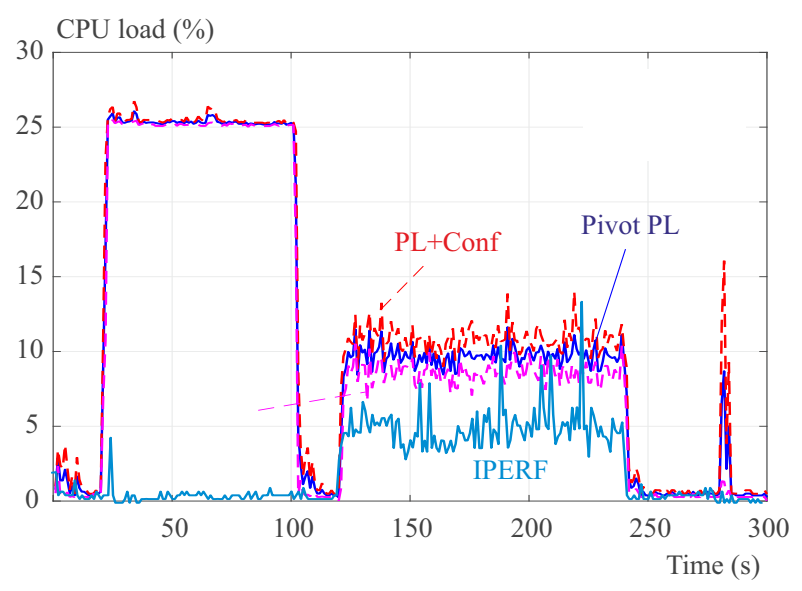

Fig. 2. Sample CPU load statistics for Snort, TCP traffic

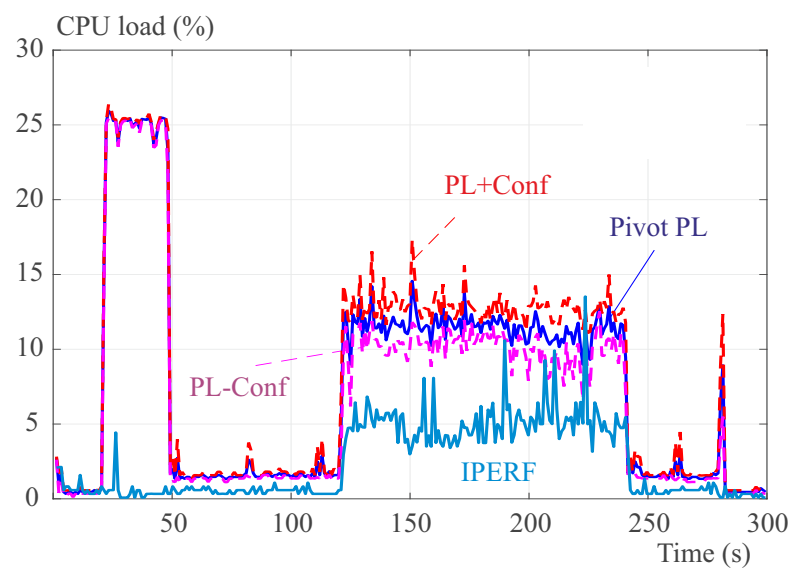

Fig. 4. Sample CPU load statistics for Suricata, TCP traffic

Table 3. Basic features of Snort and Suricata on for IPS testing

\begin{tabular}{|c|c|}
\hline Parameter & Description \\
\hline Snort Location & /home/pi \\
\hline File type & directory \\
\hline Total count of files & 2437 \\
\hline Total size of files & $111.1 \mathrm{MB}$ \\
\hline Suricata Location & /home/pi \\
\hline File type & directory \\
\hline Total count of files & 2996 \\
\hline Total size of files & 104.7 MB \\
\hline
\end{tabular}

Table 4. Specification of Raspberry Pi 1 for performance tests under data overload

\begin{tabular}{ll}
\hline Raspberry Pi 1 & Specification \\
SoC & Broadcom BCM2835 \\
CPU & Cortex A6, single core, $700 \mathrm{MHz}$ \\
RAM & $512 \mathrm{MB}$ \\
Networking & $10 / 100 \mathrm{Mbps}$ Ethernet, HDMI \\
\hline
\end{tabular}

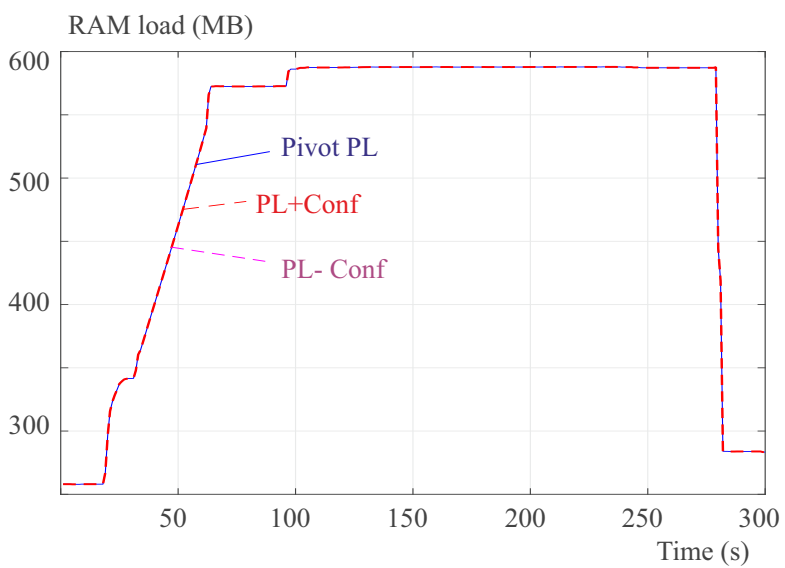

Fig. 3. Sample RAM load statistics for Snort, TCP traffic

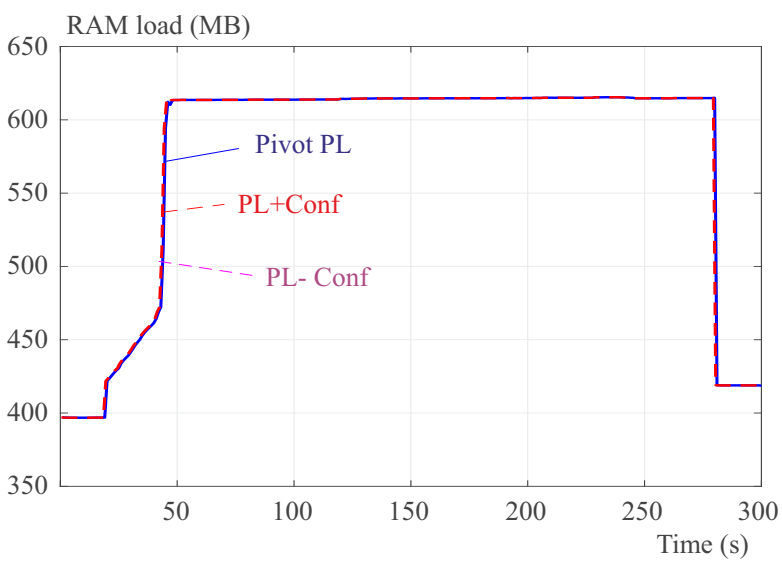

Fig. 5. Sample load statistics for Suricata, TCP traffic

The chosen options for testing purposes are in Table 11 (in Appendix).

\section{Results and discussion}

\subsection{Testing selected IPS on realistic communication} traffic for low-performance IoT devices

The diagrams in Fig. 2 to Fig. 5 show some examples of CPU load and RAM use for the above described communication network traffic, specified in Table 1 (all the number of streams, bit rates and segment sizes were tested). The statistical pivot half sums are calculated. To confirm the significance of the effect of the hardware platform evaluated, the mean difference values of CPU and RAM results were calculated for each communication network traffic. All calculated mean values are significantly different from zero, $i e$, the effect of hardware platform used is statistically significant for the observed CPU and RAM load.

Suricata does some internal cleaning as it runs. I suspect it is recovering some RAM that was initially allocated and then later not needed. During start-up, the rule 
Table 5. Maximum data rate achievable in Raspberry Pi 1 with Snort or Suricata and under data overload

\begin{tabular}{lccc}
\hline & \multicolumn{3}{c}{ Data rate in (Mbps) } \\
Run & without IPS & with Suricata & Snort \\
\hline 1 & 45 & 7.60 & 8.22 \\
2 & 45 & 7.50 & 8.28 \\
3 & 45 & 7.52 & 7.92 \\
4 & 45 & 7.63 & 8.11 \\
5 & 45 & 7.55 & 8.37 \\
6 & 45 & 7.47 & 8.29 \\
7 & 45 & 7.49 & 8.20 \\
\hline
\end{tabular}

set is loading all at once and parsing/decoding the text into all the internal structures used for the pattern matching algorithm. For this reason, it is likely that we observe the peak value at around $50 \mathrm{~s}$ time, when Snort/Suricata is loading. However, when the Iperf tool is started, the RAM consumption is already stabilized.

Network traffic has great impact on the CPU load. CPU usage depends on the type of network traffic. TCP segments load the CPU more than UDP datagrams. Smaller packets load the processor more than large packets. Finally, Snort loads the CPU up to $5 \%$ less in comparison to Suricata.

The high CPU usage during the period of running Iperf is linked to the kworker/u8+ process that utilizes kernel worker threads.

Memory usage was minimally affected by network traffic. Both Snort and Suricata consume a lot of RAM. Snort takes up more than 100 MB of memory in comparison to Suricata. RAM usage was constant during the experiment after loading Snort or Suricata, which results from the architecture of the IPS. Most of the RAM is consumed by rules. The set of rules should be limited and some parts of the IPS must be cropped.

\subsection{Performance of Snort and Suricata under data over- load}

An interesting case is the performance of Snort and Suricata under specific conditions, which can for example be CPU and RAM utilization in data overload. Suricata and Snort under overload is considered for generations of Raspberry Pi 1 (Tab. 4).

In our case, data overload is referred to maximum possible amount of data that can be processed by a microcomputer, corresponding to a specific bit rate. Setting a greater bit rate would result in transmission failure, as CPU and RAM are unable to process it. In general, adding Snort/Suricata allows lower critical bit rates.

Timeline of experiment (Raspberry Pi 1 under overload):

- 0:00 Start of experiment, SAR commands are executed.

- 0:20 Snort or Suricata is started.
- 7:05 (425s) Iperfs transmission is started.

- 9:05 (545 s) Iperfs transmission is completed.

- 9:45 (585 s) Snort or Suricata is interrupted, the system is running.

- 10:05 (605 s) End of experiment.

CPU load and RAM usage in Raspberry Pi 1 under overload condition and with Snort or Suricata used as an IPS is presented in Tab. 5 .

Raspberry Pi 1 without IPS functionality can transmit data at the rate of $45 \mathrm{Mbps}$. Loading Suricata takes 1 minute 35 seconds. Then, Raspberry Pi 1 with Suricata run as IPS can transmit at data at the rate of $7 \mathrm{Mbps}$ and for the rates above $7 \mathrm{Mbps}$, we observe degradation of transmission. Loading Snort takes from 5 minutes 55 seconds up to 6 minutes and 30 seconds. Raspberry Pi 1 with Snort can then transmit data at the rate of $8 \mathrm{Mbps}$, above this value, transmission is degraded.

Table 6. Specification of Raspberry Pi 2 for testing for performance tests under data overload

\begin{tabular}{ll}
\hline Raspberry Pi 2 & Specification \\
SoC & Broadcom BCM2836 \\
CPU & Cortex A7, quadcore, 900MHz \\
RAM & $1 \mathrm{~GB}$ \\
Networking & $10 / 100$ Mbps Ethernet, HDMI \\
\hline
\end{tabular}

Table 7. Specification of Raspberry Pi 3 for testing for performance tests under data overload

\begin{tabular}{ll}
\hline Raspberry Pi 3 & Specification \\
SoC & Broadcom BCM2837 \\
CPU & 4 ARM Cortex-A53, $1.2 \mathrm{GHz}$ \\
GPU & Broadcom VideoCore IV \\
RAM & 1 GB LPDDR2 $(900 \mathrm{MHz})$ \\
\hline
\end{tabular}

A similar experiment is carried out for Raspberry $\mathrm{Pi}$ 2 and 3 (specification in Tab. 6 and Tab. 7 respectively each generation offers more RAM and more efficient CPU handling). The same data image was used on one microSD card for all Raspberry Pi.

Timeline of experiment (Raspberry Pi 2 under overload):

- 0:00 Start of experiment, SAR commands are executed.

- 0:20 Snort or Suricata is started.

- 2:40 (160 s) Iperfs transmission is started.

- 4:40 (280 s) Iperfs transmission is completed.

- 5:20 (320 s) Snort or Suricata is interrupted, only the system is running.

- 5:40 (340s) End of experiment.

Timeline of experiment (Raspberry Pi 3 under overload):

- 0:00 Start of experiment, SAR commands are executed. 


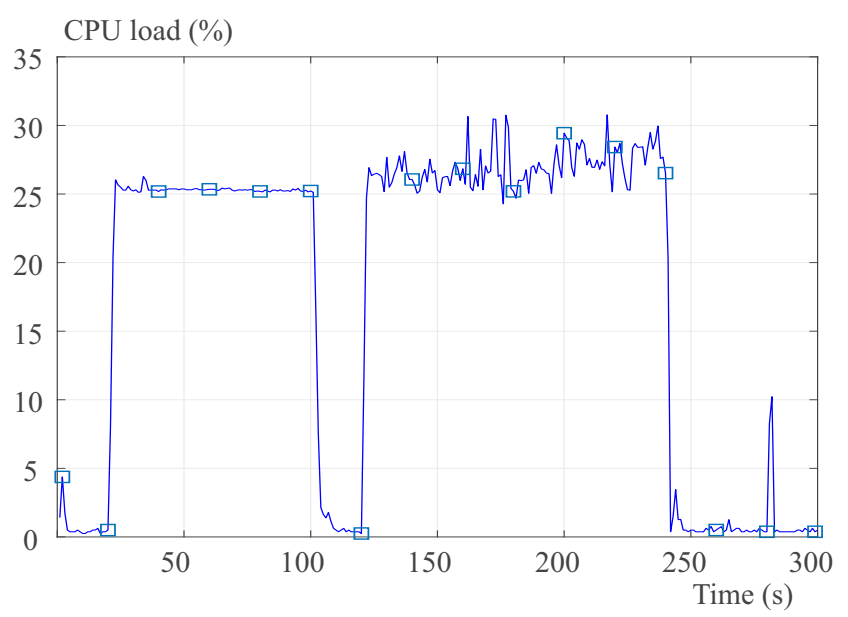

Fig. 6. CPU load statistics for Snort on Raspberry Pi 3 under overload

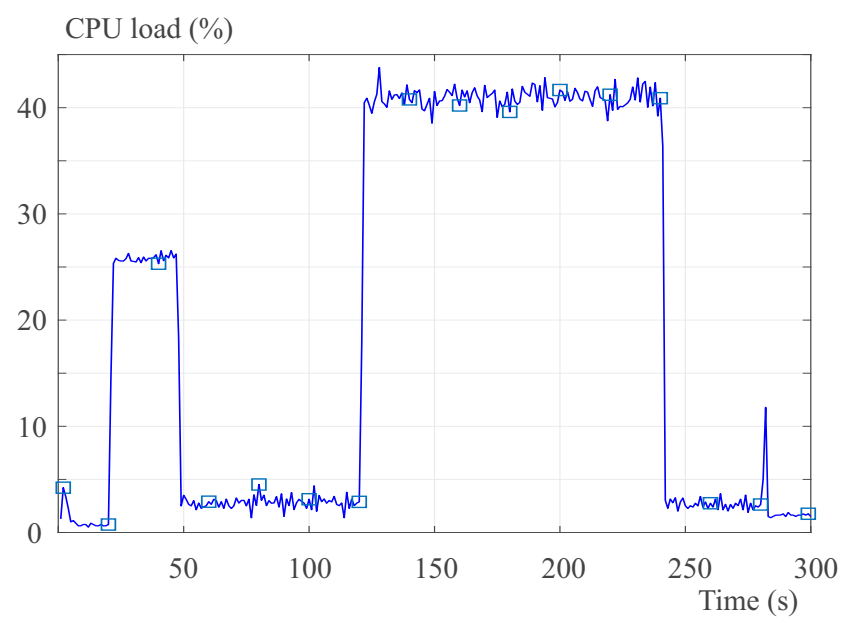

Fig. 8. CPU load statistics for Suricata on Raspberry Pi 3 under overload

- 0:20 Snort or Suricata is started.

- 2:00 (120s) Iperfs transmission is started.

- 4:00 (240 s) Iperfs transmission is completed.

- 4:40 (280 s) IPS is interrupted, only the system is running.

- 5:00 (300s) End of experiment.

Raspberry Pi 2 without IPS can transmit data at the rate of $94 \mathrm{Mbps}$, with Suricata at the rate of $67 \mathrm{Mbps}$, and with Snort at the rate of $66 \mathrm{Mbps}$. Exceeding these values can result in damaged transmission. Loading Suricata takes 46 seconds, loading Snort - 1 minute 57 seconds, the evolution of CPU and RAM usage in Raspberry Pi 2 under overload is reported in Tab. 8.

The maximum data rate in Raspberry Pi 3 without any IPS is 94 Mbps. With Suricata run as IPS, the data rate is decreased to about $93 \mathrm{Mbps}$, with Snort negligible decrease in bit rate is observed. Loading Suricata takes 27 seconds, Snort - 1 minute and 22 seconds. CPU and RAM usage in Raspberry Pi 3 under overload is shown in

\section{RAM load (MB)}

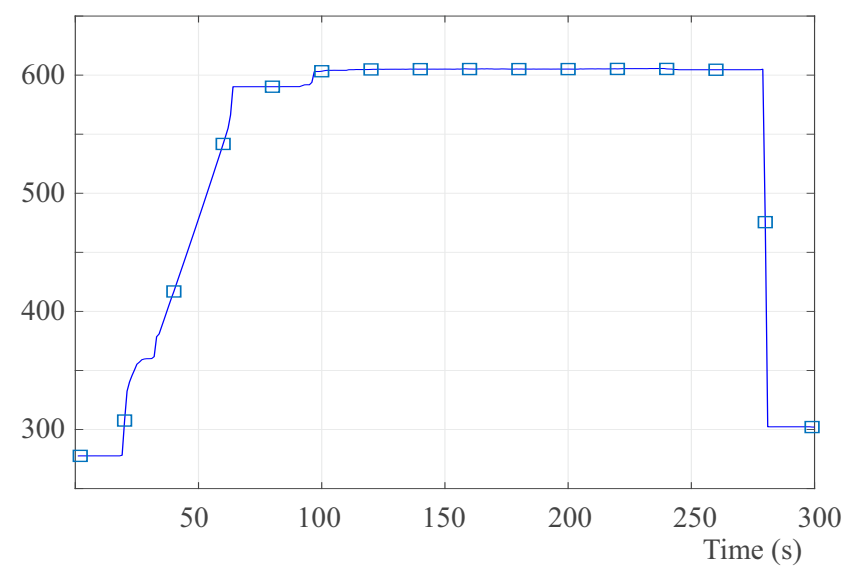

Fig. 7. RAM load statistics for Snort on Raspberry Pi 3 under overload

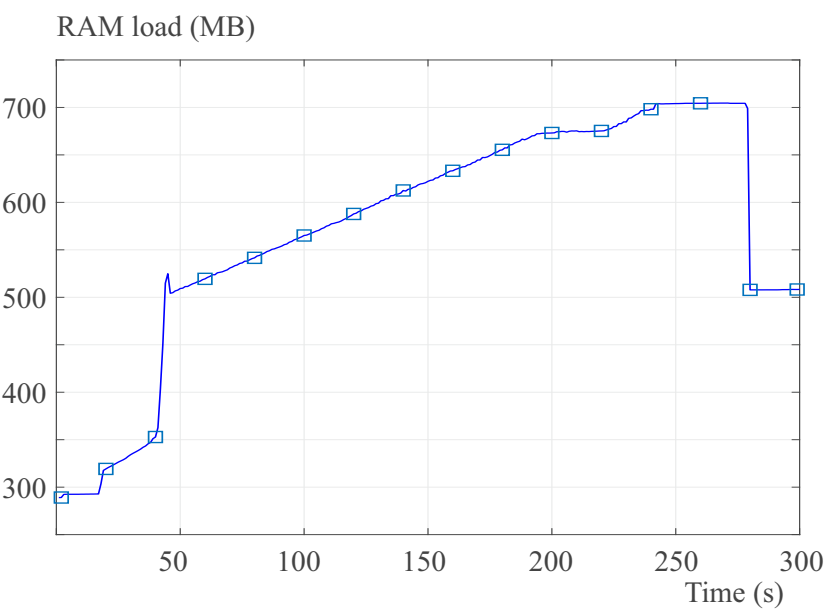

Fig. 9. RAM load statistics for Suricata on Raspberry Pi 3 under overload

Fig. 6 to Fig. 9 and in Table 9. A given IPS turns on approximately 20 seconds after the start of the experiment. It can be seen that loading Snort or Suricata results in immediate CPU consumption and slow rise in RAM usage, which then reaches a constant level independent from network traffic until IPS termination. It is followed by 2 minutes of network load test using the iperf tool, which allows to determine the adequate values of CPU load and memory consumption.

It can be noticed that CPU usage depends on network traffic. When the network load test is completed, the iperf tool is turned off. Snort/Suricata is turned off 20 seconds before the end of the experiment. The use of RAM after the experiment is completed is more than before the start of the test. RAM usage differs for particular Raspberry boards. It can be explained by different memory management. Snort requires more memory than Suricata. Suricata uses the processor more than Snort. 
Table 8. Maximum data rate achievable in Raspberry $\mathrm{Pi} 2$ with Snort or Suricata and under data overload, Data rate in run without IPS was $94 \mathrm{Mbps}$

\begin{tabular}{lcc}
\hline & \multicolumn{2}{c}{ Data rate in (Mbps) } \\
Run & Suricata & Snort \\
\hline 1 & 66.7 & 66.5 \\
2 & 68.0 & 64.5 \\
3 & 67.1 & 67.0 \\
4 & 67.5 & 66.8 \\
5 & 66.2 & 67.0 \\
6 & 66.9 & 63.0 \\
7 & 66.9 & 65.6 \\
\hline
\end{tabular}

Table 9. Maximum data rate achievable in Raspberry Pi 3 with Snort or Suricata and under data overload, Data rate in run without IPS was $94 \mathrm{Mbps}$

\begin{tabular}{lcc}
\hline & \multicolumn{2}{c}{ Data rate in (Mbps) } \\
Run & Suricata & Snort \\
\hline 1 & 93.6 & 93.3 \\
2 & 92.8 & 92.5 \\
3 & 93.3 & 92.8 \\
4 & 92.1 & 92.5 \\
5 & 93.5 & 92.9 \\
6 & 93.2 & 92.9 \\
7 & 93.3 & 93.1 \\
\hline
\end{tabular}

However, both Snort and Suricata have achieved comparable results regardless of the type of the board. One disadvantage is that Snort launches much longer than Suricata. It can be seen from the graphs that when the network load test is not run on the device, Snort uses around $5 \%$ of CPU and Suricata more than $10 \%$. The CPU usage increased for Raspberry Pi 2. Another important feature is that Snort is a single thread application and therefore launching Snort on Raspberry Pi 1 can take even 6 minutes and 30 seconds (compared to Suricata, it takes 1 minute 35 seconds). One should consider this when optimizing the solution.

\section{Conclusions}

Testing on traffic provided a clear understanding of the requirements for Snort and Suricata. Both security tools displayed different demands on system resources. One must consider specific CPU loads based on traffic, which requires reliable network traffic models. Memory use, however, can be well estimated since it depends on rules that can be known and planned.

The above results could be eligible for research, development and used in applications of Snort and Suricata as security hardware tools for IoT, in terms of their performance evaluation in relation to the level of proposed security. The results pursue a well-defined method in specifying tools for Snort/Suricata analysis for given operating systems and user applications considering whether the employment of it is justifiable.

Recommendations whether IPS should be employed or not should be aimed at the consumption of system resources. Determining hardware requirements for Snort or Suricata is conditioned by network traffic. It is not recommended to run it under overload condition, even though, it is still running, latency, for which Snort or Suricata are not optimized, is very likely to happen. For the network traffic up to $5 \mathrm{Mbps}$, Raspberry Pi 1 can be used. For traffic up to 50 Mbps, Raspberry Pi 2 can be used and above 90 Mbps Raspberry Pi 3 can be recommended as a suitable platform for IoT devices with IPS. It should be added that the ability of managing the network traffic depends on the processor performance.

During tests we used one client and one server through Iperf because the goal was to find the limit of the network traffic of connected Low Performance IoT Devices. From the perspective of a potential user who wants to update their firmware, considering more clients and one server, etc. could be more appropriate, but it depends on the chosen architecture scenario. We chose the architecture of connected IoT sensor network to Low Performance IoT Devices.

\section{Acknowledgment}

This research was funded by the Technology Agency of the Czech Republic grant number TJ01000182 and by the internal CTU grant under project SGS18/ 183/OHK3 $/ 3 \mathrm{~T} / 13$.

\section{Appendix}

Brief description of commands for testing the performance of selected IPS on Raspberry Pi Snort and Suricata and the commands for experimental testing the performance of selected IPS on Raspberry Pi Iperf tool.

\begin{tabular}{ll}
\hline Command & Function \\
\hline Snort & \\
\hline$-c$ & $\begin{array}{l}\text { configuration file } \\
\text { enables inline mode operation } \\
\text {-Q }\end{array}$ \\
-daq nfq & selects packet acquisition module NFQ \\
\hline Suricata & configuration file \\
\hline -c & runs inline of the NFQUEUE queue ID 0 \\
\hline -q 0 & reports memory utilization statistics \\
\hline Sar & reports CPU statistics \\
-r & reports CPU utilization 300 lines/s \\
sar -u 1 300 $>$ & \\
filename.txt &
\end{tabular}




\begin{tabular}{|c|c|}
\hline Command & Function \\
\hline \multicolumn{2}{|c|}{ Iperf as a server } \\
\hline$-\mathrm{s}$ & runs in server mode \\
\hline$-\mathrm{m}$ & prints TCP maximum segment size \\
\hline & $(\mathrm{MSS}=$ maximum transmission unit (MTU) TCP/IP header $)$ \\
\hline$-\mathrm{u}$ & uses UDP rather than TCP \\
\hline \multicolumn{2}{|c|}{ Iperf as a client } \\
\hline$-\mathrm{c}$ & host \\
\hline$-\mathrm{b} 5 \mathrm{M}$ & bandwidth to send at $5 \mathrm{Mbps}$ \\
\hline$-\mathrm{P} 10$ & number of simultaneous connections to make to the server \\
\hline -M 150 & attempt to set the TCP maximum segment size (MSS) to $150 \mathrm{~B}$. \\
\hline$-\mathrm{m}$ & $\begin{array}{l}\text { The MSS is usually the MTU, } 40 \text { bytes for the TCP/IP header } \\
\text { print TCP maximum segment size (MSS }=\text { MTU TCP/IP header) }\end{array}$ \\
\hline -t 120 & $\begin{array}{l}\text { time in seconds to transmit for. iPerf normally works by repeatedly } \\
\text { sending an array of len bytes for time seconds. }\end{array}$ \\
\hline$-\mathrm{u}$ & uses UDP rather than TCP \\
\hline-1 & size of datagram for UDP \\
\hline
\end{tabular}

\section{REFERENCES}

[1] M. Yassein, Q. Abuein, and S. Alasal, "Combining softwaredefined networking with Internet of Things: Survey on security performance aspects", Proceedings of the International Conference on Engineering \& MIS (ICEMIS) Monastir, Tunisia, 8-10 May 2017.

[2] A. Lupia and F. De Rango, "Performance evaluation of secure AODV with trust management under an energy aware perspective", Proceedings of the International Symposium on Performance Evaluation of Computer Telecommunication Systems Monterey, CA, USA, 6-10, 2014.

[3] C. Y. Tseung, K. P. Chow, and X. Zhang, "Anti-DDoS technique using self-learning bloom filter", Proceedings of the 2017 IEEE International Conference on Intelligence Security Informatics (ISI), Beijing China, 2017.

[4] B. Mukherjee, R. L. Neupane, and P. Calyam, "End-to-End IoT Security Middleware for Cloud-Fog Communication", Proceedings of the 2017 IEEE 4th International Conference on Cyber Security Cloud Computing (CSCloud), New York USA, 2017.

[5] G. Kalnoor and J. Agarkhed, "Pattern Matching Intrusion Detection Technique for Wireless Sensor Networks", Proceedings of the International Conference on Advances in Electrical Electronics, Information, Communication Bio-Informatics (AEEICB16), Chennai, India, 2016.

[6] G. Kalnoor and J. Agarkhed, Snort - Network Intrusion Detection \& Prevention System, Available online: https://www, snort, org (accessed on 1st August 2018).

[7] P. Singh, S. Behal, and K. Kumar, "Performance Enhancement of a Malware Detection System using Score Based Prioritization of Snort Rules", Proceedings of the 2015 International Conference on Green Computing Internet of Things (ICGCIoT), Noida India, 2016

[8] A. Sagala, "Automatic Snort IDS Rule Generation Based on Honeypot Log", Proceedings of the 2015 7th International Conference on Information Technology Electrical Engineering (ICITEE), Chiang Mai Thailand, 2015.

[9] A. Garg and P. Maheshwari, "Performance Analysis of Snortbased Intrusion Detection System", Proceedings of the 2016 3rd International Conference on Advanced Computing Communication Systems (ICACCS), Coimbatore India, 2016.
[10] N. Naik, R. Diao, and Q. Shen, "Application of Dynamic Fuzzy Rule Interpolation for Intrusion Detection: D-FRI-Snort", Proceedings of the 2016 IEEE International Conference on Fuzzy Systems (FUZZ-IEEE), Vancouver BC, Canada, 2016

[11] E. Albin and N. Rowe, "A Realistic Experimental Comparison of the Suricata Snort Intrusion-Detection Systems", Proceedings of the 2012 26th International Conference on Advanced Information Networking Applications Workshops, Fukuoka Japan, 2012.

[12] J. White, T. Fitzsimmons, and J. Matthews, "Quantitative analysis of intrusion detection systems: Snort Suricata", SPIE Defense Security Sensing Cyber Security Conference, vol. 8757 Baltimore, USA, 2013.

[13] M. Ennert, E. Chovancova, and Z. Dudlakova, "Testing of IDS model using several intrusion detection tools", Journal of Applied Mathematics Computational Mechanics, vol. 14 no. 1, pp. $55-62,2013$.

14] K. Thongkanchorn, S. Ngamsuriyaroj, and V. Visoottiviseth, "Evaluation Studies of Three Intrusion Detection Systems under Various Attacks Rule Sets", Proceedings of the 2013 IEEE International Conference of IEEE Region 10 (TENCON 2013), Xi'an China, 22-25, 2013.

[15] S. Sremath, H. Sathu, and A. Sarrafzadeh, "Free open source intrusion detection systems: a study", Proceedings of the 2015 International Conference on Machine Learning Cybernetics (ICMLC), Guangzhou China, 2015.

[16] D. Bhosale and V. Mane, "Comparative Study Analysis of Network Intrusion Detection Tools", Proceedings of the 2015 International Conference on Applied Theoretical Computing Communication Technology (iCATccT), Davangere India, 2015.

17] B. Brumen and J. Legvart, "Performance analysis of two open source intrusion detection systems", Proceedings of the 2016 39th International Convention on Information Communication Technology Electronics Microelectronics (MIPRO), Opatija, Croatia, 2016.

[18] L. Tuong and K. Kimura, "2-Step Power Scheduling with Adaptive Control Interval for Network Intrusion Detection Systems on Multicores", Proceedings of the 2016 IEEE 10th International Symposium on Embedded Multicore/Many-core Systems-on-Chip (MCSOC), Lyon France, 21-23, 2016.

[19] P. S. Horn, "Some Easy t Statistics. ", J, of the Americ. Statist. Assoc. 78, 930-936, 1983. 
[20] https://github.com/esnet/iperf.

Received 13 July 2019

Tomas Zitta was born in Prague, Czech Republic, in 1990. He received the MSc degree in electrical engineering from the Czech Technical University in Prague, Faculty of Electrical Engineering, Czech Republic in 2016. He is a PhD student in Telecommunication Engineering at the Department of Telecommunication of the Faculty of Electrical Engineering of the Czech Technical University in Prague, Czech Republic. $\mathrm{He}$ is focused on mobile application development and security in IoT systems.

Michal Lucki was born in Starachowice, Poland, in 1980. He received the MSc degree in electrical engineering from the Kielce University of Technology, Faculty of Electrical Engineering, Automatic Control and Computer Science and the $\mathrm{PhD}$ degree in telecommunication from the Czech Technical University in Prague, Faculty of Electrical Engineering, Czech Republic, in 2004 and 2007, respectively. His research interests include telecommunication, photonics and security systems. M. Lucki was awarded the Rectors Prize of the Kielce University of Technology and the Deans Prize of the Czech Technical University in Prague.

Lukas Vojtech was born in Náchod, Czech Republic, in 1977. He received MS and PhD degrees in telecommunication engineering from the Czech Technical University in Prague,
Czech Republic, in 2003 and 2010, respectively (M12). He is a leader of RFID laboratory at the Czech Technical University in Prague since 2010. His research interests are hardware prototyping and measurement especially in the field of RFID technology, and applications of electrically conductive textiles. L. Vojtech was a recipient of the CATRENE Innovation Award 2014 as a team member of the consortium of the EM4EM project, aimed at research into new methods of measuring the influence of electromagnetic fields in electric-powered vehicles.

Marek Neruda was born in Hradec Králové, Czech Republic, in 1983. He received the $\mathrm{MSc}$ and $\mathrm{PhD}$ degree in telecommunication engineering from the Czech Technical University in Prague, Faculty of Electrical Engineering, Czech Republic, in 2007 and in 2014, respectively. His research interests include RFID technology, Internet of Things and electrically conductive textile materials. M. Neruda was a recipient of the CATRENE Innovation Award 2014 as a team member of the consortium of the EM4EM project, aimed at research into new methods of measuring the influence of electromagnetic fields in electric-powered vehicles.

Lenka Mejzrova was born in Nymburk, Czech Republic, in 1983. She received the MSc degree from the Czech University of Life Sciences Prague in 2008. She started her PhD at the Department of Electroenergetics, Faculty of Electrical Engineering, Czech Technical University in Prague in 2016. Her research interests include IoT systems and smart meters. 